\title{
Contemporary surgical and non-surgical management of Peyronie's disease
}

\author{
George F. Wayne ${ }^{1}$, Billy H. Cordon ${ }^{2}$ \\ ${ }^{1}$ Mount Sinai Medical Center Division of Urology, Miami Beach, FL, USA; ${ }^{2}$ Columbia University Division of Urology at Mount Sinai Medical \\ Center, Miami Beach, FL, USA \\ Contributions: (I) Conception and design: All authors; (II) Administrative support: BH Cordon; (III) Provision of study materials or patients: All \\ authors; (IV) Collection and assembly of data: All authors; (V) Data analysis and interpretation: All authors; (VI) Manuscript writing: All authors; (VII) \\ Final approval of manuscript: All authors. \\ Correspondence to: Dr. Billy H. Cordon. 4302 Alton Road, Suite 540, Miami Beach, FL 33140, USA. Email: billy.cordon@msmc.com.
}

\begin{abstract}
Numerous treatments have been proposed for Peyronie's disease (PD). As the evidence base has expanded, the field of operative and non-operative options for patients has narrowed. Collagenase clostridium hystolyticum $(\mathrm{CCH})$ injection now comprises the medical option, and surgical possibilities entail penile plication, plaque incision/excision and grafting, and prosthesis implantation. Still, questions abound regarding the optimal approach and indication for each of these treatments. We conducted a review of literature exploring the contemporary management of PD with a particular focus on work since the last American Urologic Association's (AUA) guidelines update for PD. Recent results and discussion indicate trends toward minimal invasiveness, toward a more holistic approach to the PD patient, and away from algorithmic management, galvanized, in part, by data challenging long-held beliefs.
\end{abstract}

Keywords: Peyronie's disease (PD); penile plication; plaque incision; plaque excision; grafting, penile prosthesis; collagenase clostridium hystolyticum $(\mathrm{CCH})$

Submitted Dec 01, 2017. Accepted for publication Mar 28, 2018.

doi: $10.21037 /$ tau.2018.04.06

View this article at: http://dx.doi.org/10.21037/tau.2018.04.06

\section{Introduction}

In 1743, French Surgeon Francois De La Peyronie described a dorsal bend of the penis, attributed to irregular scarring of the shaft (1). Subsequent generations proposed a variety of surgical and non-surgical therapies for Peyronie's disease (PD), though relatively few enjoy the support of high-level evidence $(2,3)$. Today urologists are largely adopting collagenase clostridium hystolyticum $(\mathrm{CCH})$ as the non-operative treatment of choice and have chosen between plication, plaque incision or excision and grafting (PIG or PEG), and prosthetic implantation for definitive, surgical management. Nonetheless, the urologic literature since the publication of the American Urologic Association's (AUA) guidelines in 2015 (2) continues to raise fundamental questions about the best application of each of these treatment modalities. Simultaneously, research continues to elucidate our understanding of the disease, and, while alternative therapies are persistently revisited, evidencebased practice continues to narrow towards scientifically rigorous options.

\section{Background}

$\mathrm{PD}$, with a definitively diagnosed prevalence of less than $1 \%$ in American men over age 18, has long been recognized to correlate with diabetes mellitus, dupuytren's contracture, and erectile dysfunction (ED), with an age of onset in the 50s (4). Recent data suggests that patients of a lower socio-economic status may bear a greater burden of the disease, which may exhibit a geographic predilection for the Southern U.S., and a possible lead time of several years between symptoms and diagnosis $(4,5)$. Moreover, it may be widely under-diagnosed, with a probable, undiagnosed 
prevalence of $11-13 \%$ (4). These findings suggest a need for a better understanding of pathophysiology, an effective work-up-including an increased awareness of PD within the broader medical and lay communities (6) - and a mindfulness of cost.

The proposed etiology of the disease involves a broken inflammatory response to sexual micro-injury of the penile tunica albuginea. This bi-layer of largely type 1 collagen and elastin incurs microfractures, which draw inflammatory cells and cytokine release. The subsequent collagen remodeling becomes pathologic when prolonged by an over-expression of tissue metalloproteinases, various growth factors, and oxidative stress (7). Immunologic factors-autoimmune disease, HLA-B27 association, as well as relevant genes-OSF-1 (involved in osteoblast recruitment), MCP-1 (in macrophage recruitment), procollagenase 4-have been implicated and may one day be pharmaceutical targets or agents for diagnosis (8-10). Similarly under investigation are familial cases of PD chromosomal abnormalities, and epigenetic dysfunction. The latter has already been found to be successfully modifiable in animal models, for which investigators have used small interfering RNA to modulate histone acetylation and DNA activation, reducing PD plaques in rats (9).

While the microbiologic understanding of PD remains pending, macroscopic concerns-patient work-up, choice of treatment, co-morbid mental health implicationspredominate. AUA guidelines' consensus opinion recommends a work-up consisting of careful history and physical, with examination of an induced erection, with or without Doppler ultrasound, and a refrain from treatment until symptoms are present for at least 12 months and stable for 3-6 months (2). The Fourth Consultation on Sexual Medicine updated these statements to include a Grade B recommendation for the addition of the Peyronie's Disease Questionnaire (PDQ), which surveys the patient's symptomatology, bother, and pain. The Consultation also recommends that the patient's symptoms be stable and painless for 6 months before treatment, with less emphasis on the requisite time-course of symptoms prior to stability (11). Others have recommended intervention in the active phase of evolving $\mathrm{PD}$, including a proposal to investigate surgical outcomes during that stage (3). Evidently, the timedelimitation of active and stable phases appears to be highlyvariable, and as the Consultation guidelines suggest, likely is of secondary value compared to clinical course. Generally speaking, if patients have ongoing changes or pain with erection, they are regarded to be in the active phase, and intervention is delayed until quiescence of symptoms, with some buffer-time to account for an expected course of healing (e.g., 3 months).

\section{Background: penile duplex Doppler ultrasonography (PDDU)}

Additional uncertainty exists with respect to PDDU. Not all men at the authors' institution undergo this test, though it is helpful in documenting baseline ED, appropriately counseling patients and setting expectations of intervention success, and for collecting objective data for subsequent evaluation of treatment outcomes. Several authors have suggested that baseline erectile function significantly predicts post-treatment erectile function. Levine described a sample of 37 men undergoing PIG and noted subjective erection at baseline to be most valuable in predicting postoperative ED with pre-op PDDU findings statistically insignificant (12). He and Taylor later expanded this sample to include additional PIG as well as plication patients, and retrospective analysis drew similar conclusions; nonetheless they suggested retaining PDDU within the work-up algorithm (13).

Others, including Alphs in 2010, demonstrated that an objective evaluation of preoperative erectile function may be independently predictive of post-op function; specifically, her team evaluated penile artery diameter, end-diastolic velocity (EDV), peak systolic velocity (PSV) and resistive index [RI, calculated as (PSV-EDV)/PSV]. They noted an EDV consistent with venous leak as well as an $\mathrm{RI}<0.8$ to predict postoperative ED, regardless of whether patients underwent plication or PIG/PEG (14). Flores echoed these results with an analysis of $56 \mathrm{men}$, in whom baseline venous leak suggested significant reduction in penile rigidity after PIG or PEG procedures (15).

Despite the development of standard operating procedures for PDDU in 2013 (16), operator-dependent variability exists, particularly with respect to anatomic positioning of the ultrasound probe (17). When such variability is considered in conjunction with the retrospective nature of studies evaluating the utility of PDDU in PD, the value of specific measures remains unsettled. Nonetheless, PDDU addresses fundamental practical concerns.

At our institution, a separate visit for PDDU provides ample opportunity to document baseline findings, including plaque position and extent, stretched penile length, erectile rigidity, and objective measurement of curvature, in addition to hemodynamic measures. This is vital prior 
to $\mathrm{CCH}$ injections, since the penis is injected in its flaccid state. Importantly, PDDU can provide a better appreciation of the PD plaque; though palpable on physical exam as an abruptly hardened band or lump of tissue, ultrasound may delineate regions where overlying tunica albuginea or deep plaque may exist. Moreover, patients with severe ED can be better counseled about appropriate management of $\mathrm{PD}$, for instance, with IPP, while specific data-points can help to assuage patients who might perseverate over their ED. Vitally, pre-operative PDDU measurements enhance surgical review and might inform future clinical decisions, by either formal retrospective analysis or by personal reflection on the count of the treating urologist.

At the very least, the patient should undergo an inoffice evaluation with both flaccid and erect exams. Often, the plaque or bands can be palpated in the flaccid state and the intracavernosal injection should be used to measure the deformity accurately using a goniometer, to document multiplanar deformities, and to evaluate hinge defects and waste deformities.

\section{Background: emotional and economic costs}

Guiding literature also makes mention of co-morbid psychological concerns that arise in men with PD. Terrier et al. recently reviewed the relevant literature and found that upwards of $50 \%$ of patients experience depression and roughly $80 \%$ experience moderate to severe distress related to their symptoms. These experiences may vary with the physical extent of the disease, but may improve with treatment $(18,19)$, and qualitatively consist of feelings of sexual anxiety, isolation, and concerns of self-image $(19,20)$. Furthermore, a more recent analysis of data from the Investigation for Maximal Peyronie's Reduction Efficacy and Safety Studies I and II (IMPRESS I and II) (21) found that pain during intercourse and patients' sense of bother was more predictive of associated ED than the severity of physical characteristics-penile curvature, shortening (22). In sum, these results indicate the need for a psychological component to the successful treatment of a PD patient-regardless of whether he undergoes operative management or not.

As PD's complexity is better appreciated-with an emphasis on evaluation, mental health, and pathophysiologya climate of increasingly scrutinized health spending will provoke a better assessment of treatment cost. Cordon et al. considered the cost of penile plication (ranging near $\$ 2,700$ ) versus that of CCH (near \$26,000), and found that for a patient with moderate or severe curvature ( 45 or
75 degrees), no improvement in non-surgical efficacy would establish cost-equivalency based on reported data (23). Wymer et al., however, reported findings at the 2017 Sexual Medicine Society of North America (SMSNA) meeting that suggested that improved $\mathrm{CCH}$ efficacy might render it more cost-effective than operative management in select cases. Nonetheless, they found surgical management to cost $\$ 20,000$ less than $\mathrm{CCH}$ based on their institution's treatment and financial data (24). Importantly, both authors differed in their definition of success-whereas the former considered a curvature of less than 30 degrees to represent success, the latter group conceptualized it first as a $20 \%$ curvature reduction and secondarily as a recovery of penetrative sex. These works identify a vital need to consider the cost of treatment and a standard definition of what it purchases.

PD represents a complicated disease profile, demanding a multimodal understanding of its basic science and its psychosocial implications before addressing the physical penile deformity. The AUA and the Consultation on Sexual Medicine-recognize intralesional $\mathrm{CCH}$ as the leading nonsurgical intervention with an evidence basis, though its effects are modest, and recommend definitive surgical management with plication or grafting for deformities in men who are capable of penetrative sex, with prosthesis reserved for patients with intractable ED. Nonetheless, the literature contains active debate on various ways to use each of these treatments, the scope of their applications, their safety profiles, and various modifications for optimizing them.

\section{Non-surgical management}

Numerous non-surgical options for PD have been proposed. Most lack large-scale, placebo-controlled evidence and bear either theorized effect or case reports suggestive of positive function. The AUA recommends against any oral agents, which have included Vitamin E, colchicine, and PDE-5 inhibitors among others (25).

Injectable therapies for PD have been proposed since as early as the 1950s, and have included corticosteroids, interferon-alpha $2 \mathrm{~b}$, verapamil, and $\mathrm{CCH}$. Only $\mathrm{CCH}$ has withstood the scrutiny of large-scale, prospective, placebocontrolled trials. Hellstrom et al. in 2006 demonstrated that interferon-alpha $2 \mathrm{~b}$ could achieve modest improvement in objective curvature; however, erectile function was unimproved, and this agent, as well as verapamil, bears a grade $\mathrm{C}$ evidence recommendation in the AUA guidelines $(2,25,26)$. Still, many urologists continue to administer these agents in both investigational and therapeutic settings. 
$\mathrm{CCH}$ was first purified in the 1940s and utilized by dermatologists and ophthalmologists through the 1980s, when Gelbard was the first to describe its application to PD management (25). His team pursued evidence for its efficacy through two parallel phase-3 trials (IMPRESS I and II), which enrolled 832 patients over 64 U.S. centers, and described a 34\%, 17-degree, statistically significant overall improvement in curvature, compared to placebo (21). Importantly, data from these trials inform the limited use of $\mathrm{CCH}$ for patients with curvature between 30 and 90 degrees, during the stable phase of the disease, without ventral curve or hourglass deformation, and with adjunctive penile modeling $(2,25,27)$. Despite the proven success of $\mathrm{CCH}$, the stated limitations, as well as optimal timing and dosing regimens, not to mention safety concerns, have prompted significant discussion in the recent literature.

\section{CCH: intervention during the active phase}

Several authors have studied $\mathrm{CCH}$ in the active phase of PD, with positive results (28). Yang and Bennet reported a series of 49 patients receiving $\mathrm{CCH}$ injections after FDA approval. Not all received the manufacturer-recommended 4-cycle regimen, and 12 patients were injected while in the active phase of disease. They re-demonstrated overall improvement in curvature (mean 15.4 degree reduction) and erectile function. Intra-lesional injection in 12 activephase patients noted a statistically significant curvature improvement of 20 degrees, as well as a significant improvement of over $40 \%$ in the bother portion of these patients' PDQ scores. The authors suggested that this compared to a lower overall improvement in curvature for chronic-phase patients and may simply reflect a process in flux rather than a permanent effectiveness (29). Others have commented that the active-phase that Yang and Bennet described did not include the more conventional demarcation of pain resolution as a harbinger of chronicity, instead labeling as active all patients with less than 1 year of symptoms (30).

Similarly, Ziegelman et al. reported successful outcomes in a series of early-adoption CCH injections in 69 men with disease stability of at least 3 months. Though they did not disaggregate their data by active and chronic phases, their patients had exhibited PD symptoms for an average of $>25$ months and stably for $>13$ months. Nonetheless, their results allude to continued success with early or activephase injections (31).

Most recently, Nguyen et al. presented their results of
162 men treated with $\mathrm{CCH}-36$ of which were injected during active phase, defined by both presence of pain and by duration $<12$ months. This group had an average symptomatology of 8.5 months, a baseline curvature of 60.6 degrees, and no ventral curvature, but realized an average curvature improvement of 16.7 degrees and no significant differences when compared to chronic phase patients. Both chronic and active phase patients had otherwise similar IIEF scores and penile length, with no difference in adverse events. The authors posited that active phase injection may prevent collagen from forming new plaques and argued for wider adoption of acute-phase injections. Nonetheless, this group acknowledged important limitations in their decision to not utilize PDQ assessment and the variability in the length of treatment undergone-a limitation shared in all the above studies of active PD.

In total, the recently published evidence indicates that $\mathrm{CCH}$ injection during the active phase is likely safe and effective, possibly preventing a worsening of the condition. Nonetheless, further controlled, randomized trials as well as an assessment of the permanence of changes observed during active-phase disease are outstanding.

\section{CCH: variations in treatment regimen}

Other authors have sought to better clarify the dosing regimen necessary for optimal efficacy of $\mathrm{CCH}$. The manufacturer and guideline recommended regimen, based on IMPRESS I and II, consists of 4 cycles of 2 injections (every 24-72 hours), spread 6 weeks apart between cycle, with penile modeling in the interval.

Anaissie et al. in 2016 reported the results of 77 patients having undergone a variable number of cycles. Over $3 / 4$ of patients completed 3 or more cycles of $\mathrm{CCH}$ intra-lesion injection. They reported an average curvature improvement of 17 degrees (from 58 to 41 degrees), but noted that for patients with significant improvement of $>20 \%$ in curvature, the first cycle evoked the strongest response. Moreover, this response achieved sustained significance for three cycles of $\mathrm{CCH}$, but not for 4 cycles, thus casting some doubt on the need for exactly four cycles as previously recommend (32).

Ralph et al. also considered a variable injection cycle number, but focused their inquiry on the need for adjunctive penile modeling. With the addition of a homeapplied vacuum device, 15 patients in each of two treatment groups - with and without clinician-performed modelingachieved similar improvement in curvature (roughly a 23 degree improvement from a 58-59 degree baseline) and 
PDQ bother and severity domain scores (33).

Abdel Raheem and colleagues explicitly considered such a shortened protocol of $\mathrm{CCH}$ injections and included vacuum therapy as an alternative to modeling. They prospectively recruited 53 men with dorsal or lateral curvature in excess of 30 degrees and administered 3 total injections, 4 weeks apart, with patient-performed penile modeling and/or vacuum suctioning (34). At week 12, 96\% of men had a mean curvature reduction of over 17 degrees or $31 \%$ improvement, with noted significant improvement in IIEF and PDQ scores. Although these patients formed a group similar to those studied in the IMPRESS trials, the authors did not design a control arm to represent manufacturer suggested use. Nonetheless, their results illustrate the potential for a shorter, less expensive treatment course for suffering patients (35).

At the authors' institution, patients are injected either in the stable phase or active phase. In addition to the above-described studies of $\mathrm{CCH}$ in active-phase PD, the authors have encountered patient feedback suggesting a sense of distress due to the lack of intervention while waiting for stable PD. Also based on patient feedback about the logistics of injection appointments, the authors have adopted a schedule of 4 cycles of 2 injections, each 1 week apart, with 2-week sexual abstinence following each cycle.

\section{CCH: safety}

While authors continue to refine the optimal timing and dosing of $\mathrm{CCH}$ injections, others have focused on elucidating its safety profile. Hellstrom et al. recently presented a posthoc analysis of IMPRESS data, to evaluate the safety profile of continued $\mathrm{CCH}$ injection in men whose curvature drops below 30 degrees in the course of treatment. In a total of 1,493 treatment cycles, only 3 corporal ruptures were identified requiring surgical management; nonetheless, penile hematoma, swelling, and hemorrhage occurred in up to $30 \%$ of cases, with more swelling, hemorrhage, and skin pigmentation change identified in men with curvature $<30$ degrees (36).

Yafi surveyed 100 members of the SMSNA and found that over one third recalled managing corporal fracture following $\mathrm{CCH}$ injection-44\% of these adverse events occurring after a 2 -week break from sexual activity following injection. Furthermore, nearly half of these respondents did not stop anti-platelet or anticoagulant medications in their patients prior to injection (37). Hobaugh commented on these results that peri-injection considerations and preparations have been ill-defined and will need more exhaustive delineation (38). Others have commented that the high observed rate of penile fracture needs to be contextualized in a physiologic context differing from non-CCH-related fractures, and may be amenable to conservative management. Doing so may better define practitioners' and patients' understanding of the drug's safety profile (39-41).

Beilan recently added to the discussion about safety a series of 102 patients having completed a variable number of cycles of $\mathrm{CCH}$ injection; $20 \%$ incurred complications, which included a $5 \%$ corporal fracture rate (all but one requiring exploration) and a $12 \%$ hematoma rate (one requiring surgery after MRI findings suspicious for fracture)-pain and fever comprising the remaining complications (42). This group's fracture rate contrasts with the $<1 \%$ fracture rate reported by the IMPRESS trials and by Levine in 2015 (43), the $<2 \%$ fracture rate seen in Yang's group (29), and the $0 \%$ fracture rate described by Ziegelmann (31).

In sum, active research seeks to determine the best time of $\mathrm{CCH}$ intervention, its optimal dosing schedule, and its safety profile. For now, data suggests that dosing may begin in the active phase and may follow a shorter time course; the safety profile of $\mathrm{CCH}$ injection, however, needs further elucidation. Further data will improve counseling such that patients can better weigh their risks and benefits, prepare for non-surgical treatment appropriately (e.g., discontinue anticoagulants), and set expectations of possible outcomes.

\section{Treatment outcome evaluation}

Another consideration for administering $\mathrm{CCH}$, or for any treatment for PD, includes an appropriate assessment of outcome.

As mentioned, the existing literature has not agreed on a definition of successful treatment of PD. The IMPRESS trials offered a composite end-point of $20 \%$ or greater curvature improvement combined with either a 1 point improvement in PDQ bother domain or a resumption of sexual activity; others have defined a "straightened" curvature as less than 20 degrees, others as less than 30 , and others declaring the resumption of penetrative sex as indicative of success $(23,24,44,45)$. Other studies recently suggested the need for evaluating the subjective sexual experience in both the patient and his sexual partner; one group retrospectively contacted $77 \mathrm{PD}$ patients after completion of $\mathrm{CCH}$ treatment and queried them and their partners for sexual satisfaction and satisfaction 
with treatment in the hopes of identifying factors that may improve both. Although their questionnaire was novel and un-validated and less than one third of patients participated, they noted that, in addition to the resumption of intercourse as well as preserved glans sensation, a history of penile trauma-possibly representing a sexual partner's feelings of guilt-predicted greater effect of treatment (46). This highlights, again, the psychological and multifactorial treatment of $\mathrm{PD}$, and the need to be aware of it, if not to outright attempt to measure it.

Goldstein et al. also recently evaluated the female sexual partners (FSPs) of men with PD. Although they drew participants from the placebo pool of IMPRESS I and II trials, both the men and their FSPs experienced significant improvement in PDQ, PDQ-FSP (adapted for the female partner), and Female Sexual Function Index (FSFI) scores, indicating improved female sexual function (47). Despite having no actual improvement in curvature, the couple was able to experience an improved sexual experience, again reiterating the need for a holistic and inclusive evaluation of outcome in PD treatment.

$\mathrm{CCH}$ is the only non-surgical management of PD with both robust supporting data and wide adoption; nonetheless, it is expensive with outcomes and safety profile imperfectly described. The recent literature suggests a moving target in establishing the appropriate timing of this intervention, its optimal dosing regimen, and the expectations that urologists can set for their patients, let alone their patients' sexual partners. In anticipation of further studies on these topics, patients will be best served with individualized care plans.

\section{Surgical management}

Many patients might prefer or be best served by surgical management. Classically, surgery has been segmented by the degree of angulation and ED severity: plication or PIG/ PEG for patients with preserved erectile function and penile prosthesis with or without adjunctive measures for the rest. As with all treatments, practitioners often vary treatment offerings by personalized treatment plans for their patients, and the recent literature suggests numerous novel modifications to offer an even wider array of surgical options.

\section{Penile plication}

The most common surgical option for patients involves the plication-with or without incision of the corporaof the convex side of the curved penis. Originally described by Nesbit in 1965 , the procedure has taken on many modifications over the ensuing 5 decades. These most notably involve the Yachia procedure-a horizontal, Heinecke-Mikulicz closure of a vertical incision opposite the PD plaque-and the Essed-Schroeder tunical imbrication without incision (48). The latter subsequently evolved modifications to invert suture knots and also to imbricate multiple, parallel lines of tissue around the point of maximal curvature, as described in Gholami and Lue's 16-dot plication technique (49). In recent years, plication has increased in popularity and several further modifications have been proposed with an emphasis on efficiency, aesthetic outcome, and patient perception.

Unlike $\mathrm{CCH}$ injection, operative treatment begins unequivocally after the stabilization of the plaque.

The 2010 consensus panel on PD recommended surgical management for the man who "desires the most rapid and reliable result," further specifying a stable, painless disease for 6 months or longer, and suggesting that men with $>60$ degree curvature or hourglass deformity causing a hinge-effect be best served with PIG or PEG (44). Mobley et al. echoed these conditions, but also specified a penile length in excess of $13 \mathrm{~cm}$ and predicted length loss of $<20 \%$ as criteria for applying plication techniques (50). In recent years, this distinction has been challenged, with plication performed for increasingly challenging and deformed cases, and, in general, with far greater frequency than excisional/grafting techniques $(51,52)$. These authors, in fact, noted that almost three-quarters of surgical PD therapies involved no plaque manipulation and that the ratio of plication to graft procedures had tripled among American urologists (52). Dr. Morey further commented that his practice had abandoned grafting procedures, noting the safety and efficacy of plication for even the most deformed cases, as shown in Figure 1 (53).

Cordon et al. recently discussed two novel modifications to penile plication. In the Kiel's Knot Plication (Figure 2), the authors describe an imbricated approach involving 8 or more paired suture marks on either side of the shaft, along the aspect of the erect penis opposite to the plaque. A superficial incision is made between each pair of marks, allowing for a raised, partial-thickness sleeve of tunica albuginea to cover knots, which are tied in an inverted fashion and pushed under this incision. The authors also claim that closer positioning of the suture holes- to $5 \mathrm{~mm}$ apart-may contribute to preserved penile length. Overall, their series of 20 such cases demonstrated $90 \%$ patient satisfaction (51). A separate technique described contends 

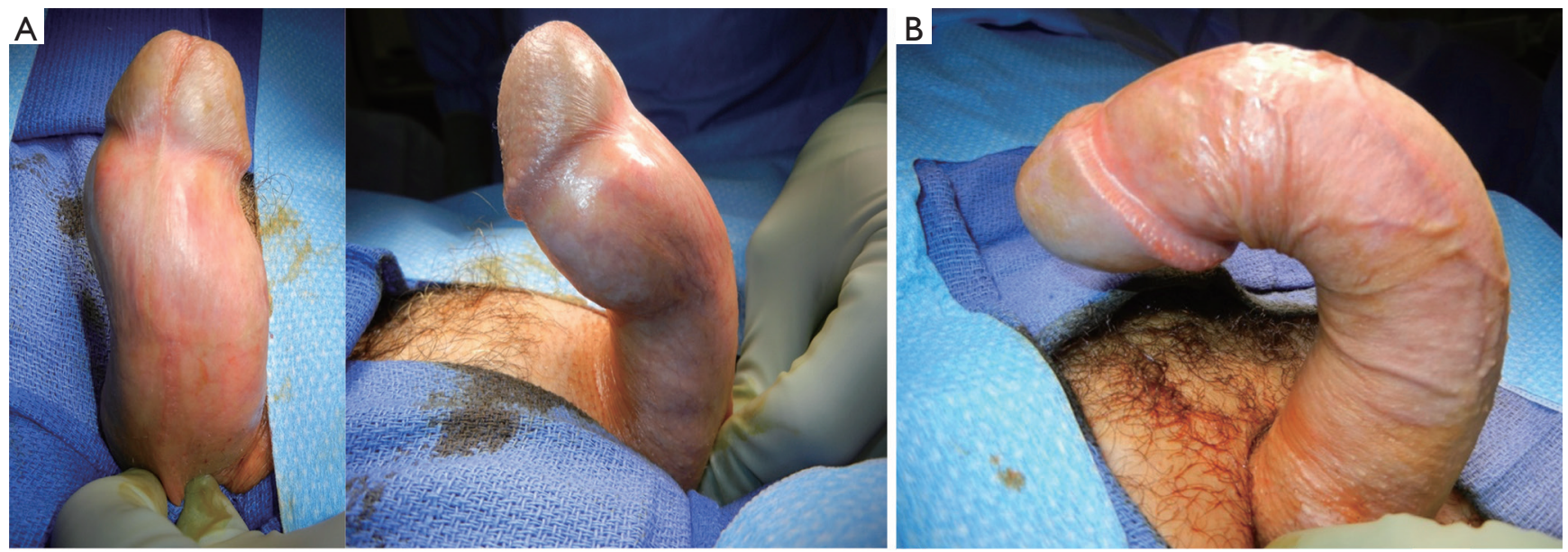

Figure 1 Challenging cases amenable to plication. (A) Multiplanar curvature caused by Peyronie's disease; (B) severe dorsal curvature in excess of 90 degrees. Both men were treated successfully with plication sutures placed to the convex aspects of each curve. Images courtesy of Allen Morey, MD.
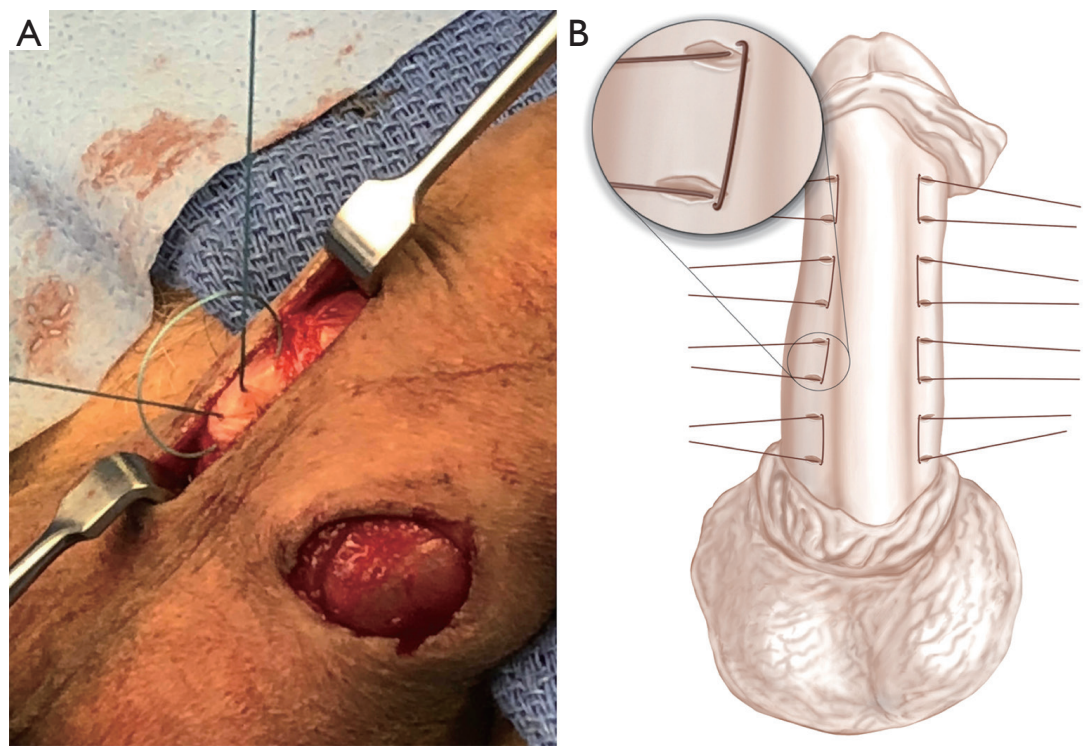

Figure 2 Examples of plication sutures. (A) Imbrication suture as described by Essed and Schroeder; (B) Kiel's Knot modification, wherein knots are directed under a partial-thickness flap of tunica albuginea, thereby addressing concerns of palpable suture knots. Illustration $\mathrm{B}$ courtesy of Daniar Osmonov, MD, PhD.

that a large dissection or degloving may be replaced with a $2-3 \mathrm{~cm}$ incision at the base of the penis with appropriate retraction and stretching allowing for operative exposure with minimal operative trauma (Figure 3). The authors of this technique present the results of 340 such minimallyinvasive plication procedures, of which 9 (2\%) required reoperation. Revision was associated with multiplanar curve, severe curvature, and new defects after surgery, as well as the possibility of under-correction due to poorly induced erections at the time of repair $(51,54)$.

Kadirov's group presented similar findings in a retrospective review of 52 patients, half of whom proceeded with minimally invasive plication, involving a $2-\mathrm{cm}$ transverse incision, while surgery for the other half of 

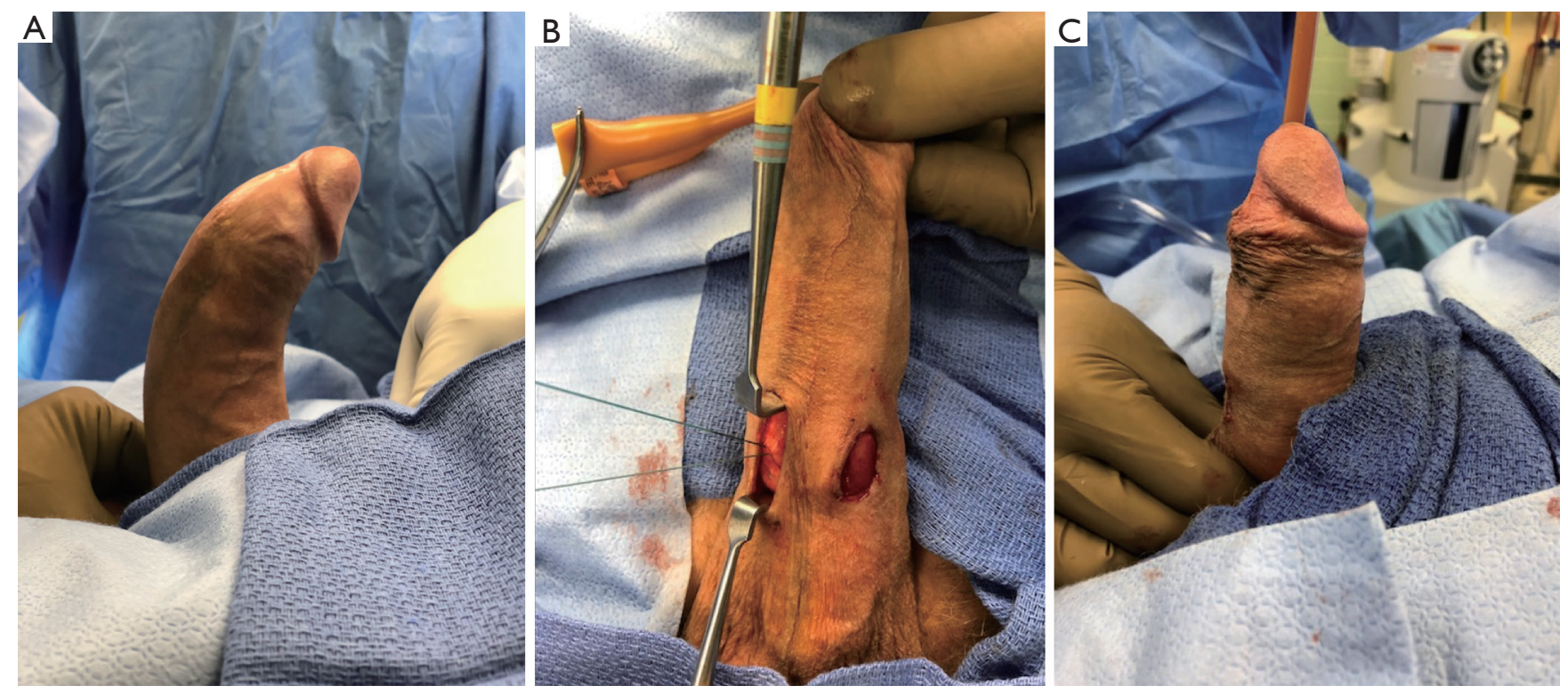

Figure 3 The minimally invasive technique is demonstrated. (A) Erection is induced at the start of the procedure and suture positions are planned; (B) 2-3 cm incisions are made near the base of the penis and retracted as needed to expose tunica for placement of plication sutures; (C) the straightened phallus is re-examined and further plication sutures are considered.

patients proceeded with de-gloving of the penis. At baseline, their curvature was measured at 36 and 38 degrees for each group (no statistically significant difference) and improved an average of 29 and 30 degrees (also not significant) with no difference seen in postoperative suture palpation, penile length, and IIEF scores (55).

Importantly, the minimally invasive approach appears to also be expeditious. Kadirov et al. reported an average operative time of nearly 62 minutes, and Cordon et al. a mean of 64 minutes, highlighting another possible source of lowered morbidity for the patient $(51,55)$.

Long described as a central fault of plication, penile shortening was less prominent than expected. Kadirov noted an average shortening length of $0 \mathrm{~cm}$ in both of his analyzed groups (55). Contrastingly, Cordon et al. had reported $35 \%$ of patients experiencing a $0.5-1.0 \mathrm{~cm}$ shortening, but which ceased to be reported after 6 months, possibly indicating a lack of subjective bother (51).

In fact, other authors have sought to explicitly analyze the patient's subjective experience. Baldini reviewed their results in 58 plication procedures (a mix of Nesbit, Yachia, and diamond-shaped repairs) for $46 \mathrm{PD}$ patients and 12 congenital curvatures. At mean follow-up of 34 months, they administered both the IIEF-5 and an un-validated, inhouse questionnaire to assess patients' subjective results; almost $70 \%$ of patients completed the questionnaires. They found that $82.5 \%$ of patients perceived at least a "clear" or more dramatic shortening of the penis, with $57.5 \%$ reporting at least some bother due to this effect. Interestingly, IIEF scores revealed moderate to severe ED in just $20 \%$ of respondents (56). Although these authors did not include a control group, their demonstrated discordance between patients' subjective feelings about the results of their procedures and their erectile function hints at a need for multifactorial evaluation of operative results, and perhaps a greater emphasis on the subjective perceptions of PD patients, especially when considered in the light of associated psychological burdens.

Plication techniques for PD have evolved significantly over the last 50 years. In recent years, plication has been afforded greater utilization, likely as part of a broader trend towards minimizing surgical invasiveness, and it has been found to have excellent outcomes in even the most difficult cases. Plication provides robust results in an expeditious, low-complication manner, with further innovation aimed at smaller incisions and more hidden sutures. Nonetheless, recent studies highlight the need for contextualizing result metrics-surgical time, presence/absence of symptoms, penile length, sensation-within the wider framework of patient experience. 


\section{Plaque incision or excision and grafting}

A grafted repair of PD curvature involves the implantation of tissue to lengthen the concave aspect of the penile shaft. Disease process stability is recommended prior to grafting (12+ months' PD, $6+$ months' stability) $(57,58)$. Historically, it has been reserved for more severe cases of PD—curvature exceeding 60 degrees or presenting with hourglass or hinge deformity, preventing penetrative sex but retaining erectile function (59). Recently, authors have specified that an Erection Hardness Score (EHS) of 3 or 4 may be necessary for good outcome in grafting procedures, as the procedure itself risks damaging cavernosal tissue and larger graft space has been linked with postoperative ED $(57,60)$. Many authors in the past have suggested that, as a procedure involving the addition of corporal tissue, it may be beneficial to patients with shorter phalluses; however, several authors have seen evidence to the contrary in follow-up $(57,61,62)$.

Numerous modalities have been proposed for corporal grafting. Hatzichristodoulou et al. reviewed these in a recent publication (63). Most procedures involve the dissection-via degloving or by midline dorsal or ventral incisions-through Buck's fascia, mobilizing the dorsolateral neurovascular bundles, and exposing the tunica albuginea, which is carefully incised over bilateral cavernosa in either $\mathrm{H}$ - or double-Y-fashion at the point of maximal curvature. This sometimes requires an ellipsoid excision of plaque. The surgeon then patches the defect with a graft of choicematerial, usually requiring suturing except in the case of collagen fleece. No gold-standard graft material has yet been identified, though many have been proposed with the goals of maximizing infection-resistance, reducing contraction, preserving erectile space, and promoting hemostasis. Materials range from autologous or donor vein, dermis, buccal or lingual mucosa, tunica, and fascia lata to bovine or porcine pericardium, small intestinal submucosa (SIS), and equine collagen fleece with several promising bio-engineered grafts under investigation (63-65).

Autologous graft harvest carries significant morbidity (scarring, pain, numbness) and additional time under anesthesia; however, patients have appeared to be satisfied with graft outcomes in short term follow-up (e.g., 1-2 years), especially when selected strictly for preserved erectile function, and despite this added morbidity (66). Contrastingly, patients followed in the long-term have demonstrated sustained decline in satisfaction (62).

Chung interviewed 46 of 86 graft patients with a minimum follow-up of 5 years (average 8 years, maximum 10) and demonstrated that initially positive results had not been durable (67). They compared patients with dermis, pericardium, and SIS grafts, which respectively had recurrent curvature in $50 \%, 13 \%$, and $14 \%$ of patientsa statistic worsened significantly since early post-operative evaluation. Moreover, two-thirds of patients used PDE5 inhibitors for ED, and between $23 \%$ and $54 \%$ of respondents perceived progressive penile shortening, with a significant drop in objective penile rigidity. Overall, $65 \%$ of respondents were dissatisfied and $61 \%$ would not undergo the procedure again. Interestingly, sexual desire and satisfaction were preserved from the early post-operative period (67). Despite confounding attrition and temporal effects, the study's results illustrate an alarming decline in treatment efficacy and patient satisfaction.

Wimpissinger et al. echoed similar findings. The authors described long-term follow-up of $>10$ years for patients undergoing autologous vein graft (dorsal penile or greater saphenous). Patients with a median baseline curvature of 90 degrees were followed for an average 13 years. $36.7 \%$ of men reported $\mathrm{ED}$, but only $17 \%$ were unable to have penetrative sex. Overall satisfaction was noted in $73 \%$ of surveys. Interestingly, the authors commented that a large subset of their population reported subjective penile shortening ( $43 \%$ of respondents), which exceeded the rate of subjective shortening among those men who went on to have additional corporal plication procedures (33\%) (57).

Other long-term follow-up series have shown more promising results of plaque incision/excision and grafting. Taylor and Levine observed retained patient satisfaction after many years, preserved erectile function, and even penile lengthening with time; interestingly, though, they saw marginally even better results with plication techniques, including on patients with baseline curvature up to 90 degrees (68).

Furthermore, PEG/PIG are on average significantly longer operations, with a reported range of $94-165$ minutes per case, varying with the graft material (63). The quickest, at roughly $50 \%$ slower than reported plication times was observed with collagen fleece, requiring no suture fixation of the graft. The mixed results of plaque incision/excision and grafting have provoked a drive towards optimizing possible approaches. Recent authors have sought to improve patient outcomes and satisfaction with multiple incisions in lieu of large grafts (60) and novel graft materials such as autologous lingual mucosa grafts, requiring 130 minutes of operating time (69), SIS grafts at 165 minutes (70), and buccal mucosa grafts at 115 minutes (71). One group 
presented the results of plaque excision and tunical reapproximation without graft, describing good results on short-term follow-up (72). Another recent publication introduced computerized mathematical modeling to better measure and prepare grafts (73). Many of these authors have reported objectively good results, though with often high surgical site complication rates (as high as $60 \%$ in the case of SIS grafts) (70), and, as described, variable patient satisfaction.

Canonically, PIG/PEG has been reserved for patients with severe deformity (i.e., hourglass), curvature exceeding 60 degrees, short phallus, and preserved erection firmness. Nonetheless, recent publications suggest that low longterm patient satisfaction, persistent concerns about penis shortening, and worsened erections may persuade the informed PD sufferer to consider alternative management, especially with the expanding scope of plication techniques and prosthesis availability. Even for severe hourglass deformity, recent comparative data suggests that patients may often be better served by a penile prosthesis, with $100 \%$ resolution of the deformity, marked improvement in SHIM score, and a smaller chance of glans hypoesthesia $(74,75)$.

\section{Penile prosthesis}

Penile prosthesis implantation represents the gold standard therapy for men with extensive deformity due to $\mathrm{PD}$ and poor erectile function. Penile implants have existed in various forms for 5 centuries, with the inflatable penile prosthesis (IPP) introduced in 1973 (76). Two manufacturers (Boston Scientific and Coloplast) currently provide the IPPs on the market (77). Since the 1990s, penile implants have proven to be a mainstay of PD management, though questions in the most recent literature persist as to the choice of implant, it's optimized placement, and the use of adjunctive measures in treating PD.

Chung recently compared American Medical Systems and Coloplast IPPs in a retrospective review; 138 men with PD, of which 50 were implanted with a Coloplast prosthesis, were seen and operated on in a 5 year span of time. Both prostheses had similar revision and explanation rates, as well as high patient satisfaction ratings; $82 \%$ would undergo the surgery again if given the option (78). This study indicates that both IPP producers make appropriate products and that the choice between them should aim to address specific patient needs and characteristics rather than be based on any expectation of objective superiority.

Patients were similarly satisfied in multiple recent studies of implantation as an adjunct to other treatments for PD. Levine and Larsen reviewed the charts of IMPRESS trials participants who subsequently required penile plication or PEG/PIG, commenting that no lasting anatomic difficulties or complications had been created by the $\mathrm{CCH}$ injections. Thus, previous non-operative management should not be viewed as a contraindication to surgery (79). Delay et al. soon followed this with a case series of 10 patients who had progressed from $\mathrm{CCH}$ to surgery for their PD. Three of those patients underwent IPP placement. The authors noted some difficulty with dissection and attributed it to inflammatory effects from $\mathrm{CCH}$, last administered less than 6 months prior. They thus recommended this as a minimum waiting period before pursuing surgery, including IPP implantation, post-CCH (80).

Other authors have recently focused discussion on novel techniques for placing the prosthesis to facilitate corporal deformity correction. Weinberg et al. presented 200 men who underwent a sub-coronal placement of a Coloplast IPP. In this technique, a circumcising incision is made and the penis is entirely degloved, allowing for concurrent corporal procedures. In this study, $24 \%$ of patients underwent corporal plication with another $22 \%$ undergoing plaque incision. The authors note that penile length was preserved for all patients seen after 6-month follow-up.

Other recent studies have focused on adjunctive intraoperative measures, which can include modeling, plication, PEG/PIG, as well as more novel techniques (81). One such innovation is the "double dorsoventral sliding technique" for adding length during prosthesis placement (Figure 4). Designed for patients with end-stage ED and severe penile shortening, this technique calls for complete degloving of the penis through subcoronal and penoscrotal incisions, followed by dissection to the tunica albuginea and mobilization of the neurovascular bundles and urethra. Each corpora is then transected in a stair-shaped incision with a central $4+\mathrm{cm}$ tunical incision on either side connecting a proximal, ventral-aspect, hemi-circumferential incision to a distal such incision on the dorsal aspect. This creates two reciprocal halves of the corpora with staggered edges, which can slide over a prosthesis cylinder and which can be fixed in place with grafted material. The first series of 28 men undergoing this repair reported $95 \%$ satisfaction with length at 3 -month follow-up, a $3.2 \mathrm{~cm}$ average gain in penile length and sustained improvement in PDQ and IIEF scores. Over half of the patients experienced transient glans hypoesthesia, but only 1 was noted to sustain this complication permanently (82). Egydio's group replicated 
A

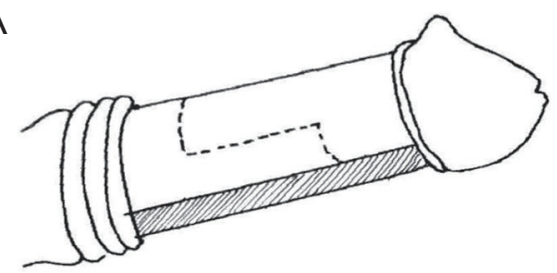

B
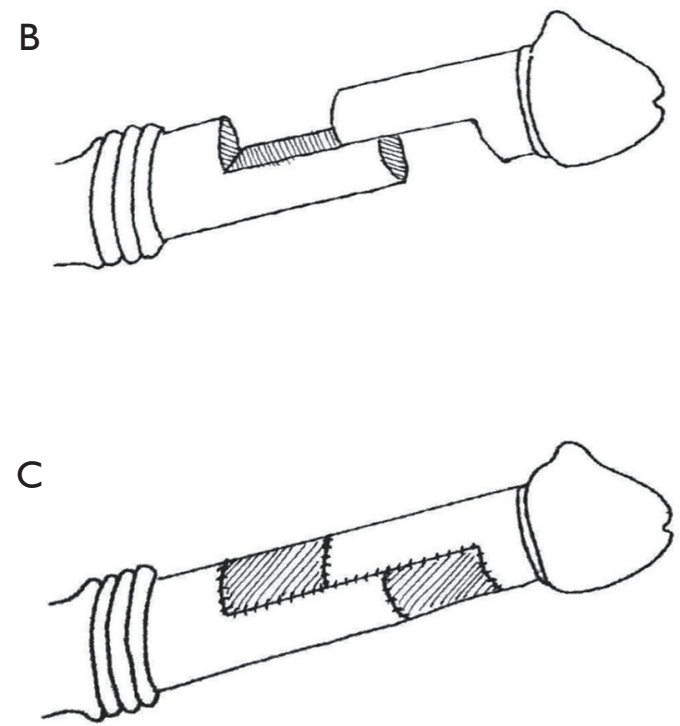

Figure 4 The double dorsoventral sliding technique is pictured. (A) The tunica albuginea is exposed by dissection through a circumcising incision; (B) after mobilizing the neurovascular bundles and the urethra, the corpora is transected in a stair-shaped fashion; (C) the staggered edges slide over a prosthesis cylinder and are sutured in place with grafted material filling the remaining gaps.

these results in 143 patients, with sustained subjective improvement in sexual experience and a $>3 \mathrm{~cm}$ length gain (83). Fang et al. later presented a variant of this procedure whereby a large ventral incision could be retracted laterally, then dorsally to expose the corpora and allow sliding incisions as well as IPP placement without degloving. They reason that degloving confers an unnecessary risk of ischemic damage to the glans and can thus be avoided with this simple and effective modification (84).

Another novel adjuvant maneuver, the "plaque scratch", involves incising a PD plaque with a hook-bladed knife from within a dilated corpora, with the intention of better freeing the plaque and allowing for more effective penile modeling in the Wilson method $(85,86)$.
A variety of surgical repairs have been evaluated for PD. Recent literature has emphasized minimal invasiveness, upended some long-held beliefs, e.g., that plication uniformly shortens and PEG/PIG lengthens the penis, and cast light on new methods for improving wellpracticed surgeries. As with non-operative intervention, the indications for each surgical approach appear to be moving targets, dependent more on individual patient needs than on demonstrated superiorities.

\section{Conclusions}

As the urologic community's understanding of PD has evolved, various treatment modalities have come and gone. A broad trend towards minimal invasiveness has coincided with the advent of $\mathrm{CCH}$ and an emerging spectrum of surgical options. Although $\mathrm{CCH}$ has shown tremendous promise, its exact timing and dosing have remained under review, with data to support shorter and earlier injection regimens. Nonetheless, many authors question its cost as well as the magnitude of its effect. On the surgical front, an emphasis on smaller incisions, faster operating times, and better aesthetic outcomes prevails. Still, there often exists a gap between patients' subjective assessments of outcomes and objective measures, with long-term dissatisfaction and concerns of penile shortening commonly seen. Simultaneously, a widening scope for plication techniques (e.g., to treat greater and more complex deformities) and an awareness of IPP may erode the pool of patients who would otherwise have pursued PIG /PEG treatments. PD represents a spectrum of patients with variable deformity, erectile function, goals of care, and self-perception-all of which should be addressed by the treating urologist, and all of which is unlikely amenable to a modular treatment algorithm. As the disease is better understood and its available treatments further optimized, management will likely remain fluid and encompass aspects of multiple treatment modalities to best serve each individual patient.

\section{Acknowledgements}

None.

\section{Footnote}

Conflicts of Interest: The authors have no conflicts of interest to declare. 


\section{References}

1. Dunsmuir WD, Kirby RS. Francois de LaPeyronie (19781747): the man and the disease he described. Br J Urol 1996;78:613-22.

2. American Urological Association - Peyronie's Disease [Internet]. [cited 2017 Nov 5]. Available online: http:// www.auanet.org/guidelines/peyronies-disease-(2015)

3. Terlecki RP, Rasper AM. Data vs Dogma in Peyronie's Disease. Int Braz J Urol 2016;42:1058-61.

4. Stuntz M, Perlaky A, des Vignes F, et al. The Prevalence of Peyronie's Disease in the United States: A PopulationBased Study. PLoS One 2016;11:e0150157.

5. Shindel AW, Sweet G, Thieu W, et al. Prevalence of Peyronie's Disease-Like Symptoms in Men Presenting With Dupuytren Contractures. Sex Med 2017;5:e135-41.

6. Love C, Katz DJ, Chung E, et al. Peyronie's disease - Watch out for the bend. Aust Fam Physician 2017;46:655-9.

7. Sherer BA, Levine LA. Contemporary Review of Treatment Options for Peyronie's Disease. Urology 2016;95:16-24.

8. Campbell J, Alzubaidi R. Understanding the cellular basis and pathophysiology of Peyronie's disease to optimize treatment for erectile dysfunction. Transl Androl Urol 2017;6:46-59.

9. Herati AS, Pastuszak AW. The Genetic Basis of Peyronie Disease: A Review. Sex Med Rev 2016;4:85-94.

10. Paulis G, Romano G, Paulis L, et al. Recent Pathophysiological Aspects of Peyronie's Disease: Role of Free Radicals, Rationale, and Therapeutic Implications for Antioxidant Treatment-Literature Review. Adv Urol 2017;2017:4653512.

11. Chung E, Ralph D, Kagioglu A, et al. Evidence-Based Management Guidelines on Peyronie's Disease. J Sex Med 2016;13:905-23.

12. Levine LA, Greenfield JM, Estrada CR. Erectile dysfunction following surgical correction of Peyronie's disease and a pilot study of the use of sildenafil citrate rehabilitation for postoperative erectile dysfunction. J Sex Med 2005;2:241-7.

13. Taylor FL, Abern MR, Levine LA. Predicting Erectile Dysfunction Following Surgical Correction of Peyronie's Disease without Inflatable Penile Prosthesis Placement: Vascular Assessment and Preoperative Risk Factors. J Sex Med 2012;9:296-301.

14. Alphs HH, Navai N, Köhler TS, et al. Preoperative Clinical and Diagnostic Characteristics of Patients Who Require Delayed IPP after Primary Peyronies Repair. J
Sex Med 2010;7:1262-8.

15. Flores S, Choi J, Alex B, et al. Erectile Dysfunction after Plaque Incision and Grafting: Short-term Assessment of Incidence and Predictors. J Sex Med 2011;8:2031-7.

16. Sikka SC, Hellstrom WJ, Brock G, et al. Standardization of Vascular Assessment of Erectile Dysfunction. J Sex Med 2013;10:120-9.

17. Pagano MJ, Stahl PJ. Variation in Penile Hemodynamics by Anatomic Location of Cavernosal Artery Imaging in Penile Duplex Doppler Ultrasound. J Sex Med 2015;12:1911-9.

18. Hellstrom WJ, Feldman RA, Coyne KS, et al. Self-report and Clinical Response to Peyronie's Disease Treatment: Peyronie's Disease Questionnaire Results From 2 Large Double-Blind, Randomized, Placebo-Controlled Phase 3 Studies. Urology 2015;86:291-8.

19. Terrier JE, Nelson CJ. Psychological aspects of Peyronie's disease. Transl Androl Urol 2016;5:290-5.

20. Rosen R, Catania J, Lue T, et al. Impact of Peyronie's disease on sexual and psychosocial functioning: qualitative findings in patients and controls. J Sex Med 2008;5:1977-84.

21. Gelbard M, Goldstein I, Hellstrom WJ, et al. Clinical efficacy, safety and tolerability of collagenase clostridium histolyticum for the treatment of peyronie disease in 2 large double-blind, randomized, placebo controlled phase 3 studies. J Urol 2013;190:199-207.

22. Serefoglu EC, Smith TM, Kaufman GJ, et al. Factors Associated With Erectile Dysfunction and the Peyronie's Disease Questionnaire in Patients With Peyronie Disease. Urology 2017;107:155-60.

23. Cordon BH, Hofer MD, Hutchinson RC, et al. Superior Cost Effectiveness of Penile Plication vs Intralesional Collagenase Injection for Treatment of Peyronie's Disease Deformities. Urol Pract 2017;4:118-25.

24. Wymer K, Ziegelmann MJ, Trost LW. Collagenase Clostridium Histolyticum is a Cost-effective Treatment for Peyronie's Disease in Appropriately Selected Patients [Internet]. [cited 2017 Nov 26]. Available online: http:// smsna.org/V1/index.php?option=com_content\&view=artic le\&id=419\&abstract_id=166

25. Yang KK, Bennett N. The History of Collagenase Clostridium Histolyticum. Sex Med Rev 2015;3:289-97.

26. Hellstrom WJ, Kendirci M, Matern R, et al. Single-blind, multicenter, placebo controlled, parallel study to assess the safety and efficacy of intralesional interferon alpha-2B for minimally invasive treatment for Peyronie's disease. J Urol 2006;176:394-8.

27. Ahn ST, Moon DG. Collagenase clostridium hystolyticum 
in the management of Peyronie's disease. Transl Androl Urol 2017;6:305-7.

28. Gabrielson AT, Spitz JT, Hellstrom WJ. Collagenase Clostridium Histolyticum in the Treatment of Urologic Disease: Current and Future Impact. Sex Med Rev 2018;6:143-56.

29. Yang KK, Bennett N. Peyronie's Disease and Injectable Collagenase Clostridium histolyticum: Safety, Efficacy, and Improvements in Subjective Symptoms. Urology 2016;94:143-7.

30. Aliperti LA, Mehta A. Expanding the role of injectable collagenase clostridium histolyticum for the treatment of active phase Peyronie's disease. Transl Androl Urol 2017;6:114-5.

31. Ziegelmann MJ, Viers BR, McAlvany KL, et al. Restoration of Penile Function and Patient Satisfaction with Intralesional Collagenase Clostridium Histolyticum Injection for Peyronie's Disease. J Urol 2016;195:1051-6.

32. Anaissie J, Yafi FA, DeLay KJ, et al. Impact of Number of Cycles of Collagenase Clostridium Histolyticum on Outcomes in Patients With Peyronie's Disease. Urology 2017;100:125-30.

33. Ralph DJ, Abdel Raheem A, Liu G. Treatment of Peyronie's Disease With Collagenase Clostridium histolyticum and Vacuum Therapy: A Randomized, OpenLabel Pilot Study. J Sex Med 2017;14:1430-7.

34. Abdel Raheem A, Johnson M, Abdel-Raheem T, et al. Collagenase Clostridium histolyticum in the Treatment of Peyronie's Disease-A Review of the Literature and a New Modified Protocol. Sex Med Rev 2017;5:529-35.

35. Abdel Raheem A, Capece M, Kalejaiye O, et al. Safety and effectiveness of collagenase clostridium histolyticum in the treatment of Peyronie's disease using a new modified shortened protocol. BJU Int 2017;120:717-23.

36. Hellstrom WJ, Tan RB, Liu G. Safety Profile of Collagenase Clostridium Histolyticum Stratified by Degree of Penile Curvature in Patients With Peyronie Disease. Urology 2017;106:237.e9-14.

37. Yafi FA, Anaissie J, Zurawin J, et al. Results of SMSNA Survey Regarding Complications Following Intralesional Injection Therapy With Collagenase Clostridium Histolyticum for Peyronie's Disease. J Sex Med 2016;13:684-9.

38. Hobaugh C, Pastuszak AW. Serious complications of collagenase clostridium histiolyticum injection for Peyronie's disease: more than meets the eye! Transl Androl Urol 2017;6:320-1.

39. Kahn B, Bennett N. Re: Results of SMSNA survey regarding complications following intralesional injection therapy with collagenase clostridium histolyticum for Peyronie's disease. Transl Androl Urol 2017;6:111-3.

40. Levine LA. Complications and other concerns with intralesional injection therapy with collagenase clostridium histolyticum for Peyronie's disease. Transl Androl Urol 2017;6:120-2.

41. Yan S, Yap T, Minhas S. Collagenase clostridium histolyticum intralesional injections for the treatment of Peyronie's disease: a safety profile. Transl Androl Urol 2017;6:123-6.

42. Beilan JA, Wallen JJ, Baumgarten AS, et al. Intralesional Injection of Collagenase Clostridium histolyticum May Increase the Risk of Late-Onset Penile Fracture. Sex Med Rev 2018;6:272-278.

43. Levine LA, Cuzin B, Mark S, et al. Clinical Safety and Effectiveness of Collagenase Clostridium Histolyticum Injection in Patients with Peyronie's Disease: A Phase 3 Open-Label Study. J Sex Med 2015;12:248-58.

44. Ralph D, Gonzalez-Cadavid N, Mirone V, et al. The Management of Peyronie's Disease: Evidence-based 2010 Guidelines. J Sex Med 2010;7:2359-74.

45. Levine LA, Larsen SM. Surgery for Peyronie's disease. Asian J Androl 2013;15:27-34.

46. Anaissie J, Yafi FA, Traore EJ, et al. Survey of patient and partner satisfaction following collagenase Clostridium histolyticum treatment for Peyronie's disease. Andrology 2017;5:274-7.

47. Goldstein I, Knoll LD, Lipshultz LI, et al. Changes in the Effects of Peyronie's Disease After Treatment With Collagenase Clostridium histolyticum: Male Patients and Their Female Partners. Sex Med 2017;5:e124-30.

48. Chen R, McCraw C, Lewis R. Plication proceduresexcisional and incisional corporoplasty and imbrication for Peyronie's disease. Transl Androl Urol 2016;5:318-33.

49. Gholami SS, Lue TF. Correction of Penile Curvature Using The 16-DOT Plication Technique: A Review of 132 Patients. J Urol 2002;167:2066-9.

50. Mobley EM, Fuchs ME, Myers JB, et al. Update on plication procedures for Peyronie's disease and other penile deformities. Ther Adv Urol 2012;4:335-46.

51. Cordon BH, Osmonov D, Hatzichristodoulou G, et al. Peyronie's penile plication. Transl Androl Urol 2017;6:639-44.

52. Oberlin DT, Liu JS, Hofer MD, et al. An Analysis of Case Logs From American Urologists in the Treatment of Peyronie's Disease. Urology 2016;87:205-9.

53. Morey AF. Re: An Analysis of Case Logs from American 
Urologists in the Treatment of Peyronie's Disease. J Urol 2016;196:1519.

54. Cordon BH, Sundaram V, Hofer MD, et al. Penile Plication as Salvage Strategy for Refractory Peyronie's Disease Deformities. Urol Pract 2017;4:149-54.

55. Kadirov R, Coskun B, Kaygisiz O, et al. Penile Plication With or Without Degloving of the Penis Results in Similar Outcomes. Sex Med 2017;5:e142-7.

56. Baldini A, Morel-Journel N, Paparel P, et al. Patientreported long-term sexual outcomes following plication surgery for penile curvature: A retrospective 58-patient study. Prog Urol 2017;27:10-6.

57. Wimpissinger F, Parnham A, Gutjahr G, et al. 10 Years' Plaque Incision and Vein Grafting for Peyronie's Disease: Does Time Matter? J Sex Med 2016;13:120-8.

58. Hatzichristodoulou G. Grafting techniques for Peyronie's disease. Transl Androl Urol 2016;5:334-41.

59. Mulhall J, Anderson M, Parker M. A Surgical Algorithm for Men with Combined Peyronie's Disease and Erectile Dysfunction: Functional and Satisfaction Outcomes. J Sex Med 2005;2:132-8.

60. Hatzichristodoulou G, Tsambarlis P, Kubler H, et al. Peyronie's graft surgery-tips and tricks from the masters in andrologic surgery. Transl Androl Urol 2017;6:645-56.

61. Kadioglu A, Akman T, Sanli O, et al. Surgical treatment of Peyronie's disease: a critical analysis. Eur Urol 2006;50:235-48.

62. Kalsi J, Minhas S, Christopher N, et al. The results of plaque incision and venous grafting (Lue procedure) to correct the penile deformity of Peyronie's disease. BJU Int 2005;95:1029-33.

63. Hatzichristodoulou G, Osmonov D, Kubler H, et al. Contemporary Review of Grafting Techniques for the Surgical Treatment of Peyronie's Disease. Sex Med Rev 2017;5:544-52.

64. Imbeault A, Bernard G, Ouellet G, et al. Surgical Option for the Correction of Peyronie's Disease: An Autologous Tissue-Engineered Endothelialized Graft. J Sex Med 2011;8:3227-35.

65. Ferretti L, Giuliani M, Bessède T, et al. Tissue Engineering for Penile Surgery: Comparative Study of Noncellular and Cell-Seeded Synthetic Grafts for Tunica Albuginea Replacement. J Sex Med 2012;9:625-31.

66. Sansalone S, Garaffa G, Djinovic R, et al. Long-term results of the surgical treatment of Peyronie's disease with Egydio's technique: a European multicentre study. Asian J Androl 2011;13:842-5.

67. Chung E, Clendinning E, Lessard L, et al. Five-Year
Follow-Up of Peyronie's Graft Surgery: Outcomes and Patient Satisfaction. J Sex Med 2011;8:594-600.

68. Taylor FL, Levine LA. Surgical correction of Peyronie's disease via tunica albuginea plication or partial plaque excision with pericardial graft: long-term follow up. J Sex Med 2008;5:2221-8; discussion 2229-2230.

69. Salem EA, Elkady EH, Sakr A, et al. Lingual Mucosal Graft in Treatment of Peyronie Disease. Urology 2014;84:1374-7.

70. Cosentino M, Kanashiro A, Vives A, et al. Surgical treatment of Peyronie's disease with small intestinal submucosa graft patch. Int J Impot Res 2016;28:106.

71. Fabiani A, Servi L, Fioretti F, et al. Buccal mucosa is a promising graft in Peyronie's disease surgery. Our experience and a brief literature review on autologous grafting materials. Arch Ital Urol Androl 2016;88:115-21.

72. Ahmadnia H, Kamalati A, Younesi Rostami M, et al. The Therapeutic Effects of Intracavernosal Plaque Excision in Peyronie's Disease: A None Grafting or Tunical Excising Procedure. World J Plast Surg 2016;5:62-6.

73. Miranda AF, Sampaio FJ. Practical Computerized Solution for Incision and Grafting in Peyronie's Disease. Sex Med 2016;4:e73-82.

74. Yafi FA, Hatzichristodoulou G, Wang J, et al. Outcomes of Surgical Management of Men With Peyronie's Disease With Hourglass Deformity. Urology 2016;91:119-23.

75. Morey AF. Re: Outcomes of Surgical Management of Men with Peyronie's Disease with Hourglass Deformity. J Urol 2016;196:1692-3.

76. Scott FB, Byrd GJ, Karacan I, et al. Erectile Impotence Treated With an Implantable, Inflatable Prosthesis: Five Years of Clinical Experience. JAMA 1979;241:2609-12.

77. Levine LA, Becher E, Bella A, et al. Penile Prosthesis Surgery: Current Recommendations From the International Consultation on Sexual Medicine. J Sex Med 2016;13:489-518.

78. Chung E, Solomon M, DeYoung L, et al. Comparison between AMS 700TM CX and ColoplastTM Titan inflatable penile prosthesis for Peyronie's disease treatment and remodeling: clinical outcomes and patient satisfaction. J Sex Med 2013;10:2855-60.

79. Levine LA, Larsen SM. Surgical Correction of Persistent Peyronie's Disease Following Collagenase Clostridium Histolyticum Treatment. J Sex Med 2015;12:259-64.

80. DeLay K, Diao L, Nguyen HM, et al. Successful Treatment of Residual Curvature in Peyronie Disease in Men Previously Treated With Intralesional Collagenase Clostridium Histolyticum. Urology 2017;110:110-3. 
81. Berookhim BM, Karpman E, Carrion R. Adjuvant Maneuvers for Residual Curvature Correction During Penile Prosthesis Implantation in Men with Peyronie's Disease. J Sex Med 2015;12:449-54.

82. Rolle L, Falcone M, Ceruti C, et al. A prospective multicentric international study on the surgical outcomes and patients' satisfaction rates of the 'sliding' technique for end-stage Peyronie's disease with severe shortening of the penis and erectile dysfunction. BJU Int 2016;117:814-20.

83. Egydio PH, Kuehhas FE. Penile lengthening and

Cite this article as: Wayne GF, Cordon BH. Contemporary surgical and non-surgical management of Peyronie's disease. Transl Androl Urol 2018;7(4):603-617. doi: 10.21037/ tau.2018.04.06 widening without grafting according to a modified 'sliding' technique. BJU Int 2015;116:965-72.

84. Fang A, Wang R. Nondegloving technique for Peyronie's disease with penile prosthesis implantation and double dorsal-ventral patch graft. Asian J Androl 2018;20:90-2.

85. Wilson SK, Delk JR. A New Treatment for Peyronie's Disease: Modeling the Penis Over an Inflatable Penile Prosthesis. J Urol 1994;152:1121-3.

86. Perito P, Wilson SK. The Peyronie's Plaque "Scratch": An Adjunct to Modeling. J Sex Med 2013;10:1194-7. 